\title{
Landauer's Principle of Minimum Energy might place limits on the detectability of gravitons of certain mass
}

\author{
${ }^{1}$ Ioannis Haranas, ${ }^{2}$ Ioannis Gkigkitzis ${ }^{3}$ Kristin Cobbett, and ${ }^{4}$ Ryan Gauthier \\ ${ }^{1,3,4}$ Dept. of Physics and Computer Science, Wilfrid Laurier University, \\ Waterloo, ON, N2L 3C5, \\ Canada
}

${ }^{2}$ NOVA, Department of Mathematics, 8333 Little River Turnpike Annandale, VA 22003

USA

\begin{abstract}
According to Landauer's principle, the energy of a particle may be used to record or erase $N$ number of information bits within the thermal bath. The maximum number of information $N$ recorded by the particle in the heat bath is found to be inversely proportional to its temperature $T$. If at least one bit of information is transferred from the particle to the medium, then the particle might exchange information with the medium. Therefore for at least one bit of information, the limiting mass that can carry or transform information assuming a temperature $T=2.73 \mathrm{~K}$ is equal to $m=4.718 \times 10^{-40} \mathrm{~kg}$ which is many orders of magnitude smaller that the masse of most of today's elementary particles. Next, using the corresponding temperature of a graviton relic and assuming at least one bit of information the corresponding graviton mass is calculated and from that, a relation for the number of information $N$ carried by a graviton as a function of the graviton mass $m_{g r}$ is derived. Furthermore, the range of information number contained in a graviton is also calculated for the given range of graviton mass as given by Nieto and Goldhaber, from which we find that the range of the graviton is inversely proportional to the information number $N$. Finally, treating the gravitons as harmonic oscillators in an enclosure of size $R$ we derive the range of a graviton as a function of the cosmological parameters in the present era.
\end{abstract}

Key words: Landauer Principle, Landauer limit, graviton, graviton mass, entropy, information,

\section{Introduction}

The existence of any physical systems presupposes the registration of information just because it exists. Dynamically evolved systems not only process but they also transform information. In this case is the laws of physics determine the precise amount of information that a system can register or process or transfer as well as the exact number of logical operation that the system can perform. In Landauer's original paper (1988), the author went on making the statement that information is physical but at the same time all this information is registered and processed by physical systems. Furthermore, the laws of physics that describe a certain system can involve information and information processing. On the other hand, Landauer's principle is a physical principle that defines the lower possible theoretical limit of energy consumption during a computation. Landauer postulated that any "logically" irreversible manipulation of information, such as the erasure of a bit or the merging of two computation paths, must be accompanied by 
a corresponding entropy increase of non-information bearing degrees of freedom in the information processing apparatus or its environment" (Bennett 2003). In other words, we can say that Landauer (1961) simply postulated that information being physical has an energy equivalent, it must also obey the laws of physics and in particular the laws of the thermodynamics. At this point, we must also say that even though non-equilibrium extensions as well as quantum extensions to the Landauer principle have not been considered yet. Furthermore, Landauer's principle is a physical principle pertaining to the lower theoretical limit of energy consumption of a computation. He also postulated that any "logically irreversible manipulation of information, such as the erasure of a bit or the merging of two computation paths, must be accompanied by a corresponding entropy increase in non-information bearing degrees of freedom of the information processing apparatus or its environment" (Bennett 2003). Landauer's principle asserts that there is a minimum possible amount of energy required to change one bit of information, known as the Landauer's limit, and it is equal to:

$$
E_{\text {min }}=k_{B} T \ln 2,
$$

where $k_{B}=1.38 \times 10^{-23} \mathrm{~J} / \mathrm{K}$ is Boltzmann's constant, and $T$ is the temperature of the circuit. Therefore, Landauer's energy formula is written in terms of the information number $N \neq 1$ in the following way (Haranas and Gkigkitzis 2013):

$$
E_{\min }=N k_{B} T \ln 2
$$

In a recent paper by Vopson (2019) the author postulates the idea of a new principle of mass-energyinformation equivalence. He simply proposes that a bit of information it's not physical as it has already been proposed but there is actually a certain mass associated with it where the information its stored. For example, at room temperature $T=300 \mathrm{~K}$ the mass corresponding to one bit of information is equal to $m_{N=1 b i t}=3.190 \times 10^{-38} \mathrm{~kg}$.

\section{The theory}

In a private communication paper by Bormashenko (2019), the author postulates and considers a particle with energy $E$ in contact but not necessarily in thermal equilibrium with the thermal bath at temperature $T$. According to Landauer's principle, the energy of a particle may be used to record or erase $N$ number of information bits within the thermal bath. The maximum number of information recorded by the particle in the heat bath is equal to:

$$
N_{\text {max }}=\frac{m c^{2}}{k_{B} T \ln 2} .
$$


Equation 3 can be considered as the maximum number of information contents in bits of a particle at rest (Bormanshenko, 2019). If at least one bit of information is transferred from the particle to the medium, then the particle might exchange information with the medium. In this case we can write that $N_{\max }$ $\geq 1$ should be true. Following Bormanshenko, ibid (2019) using Eq. 3 we obtain the following relation for the mass of the particle we have that:

$$
m_{0} \geq \frac{k_{B} T \ln 2}{c^{2}}
$$

To get an idea with of the limiting mass that can carry or transform information to the medium following (Bormanshenko (2019) let us assume the temperature to be that of the background radiation of the universe namely $T=2.725 \mathrm{~K}$ we obtain that:

$$
m_{0} \geq \frac{\left(1.38 \times 10^{-23} \mathrm{~J} / \mathrm{K}\right)(2.725 \mathrm{~K})}{\left(9 \times 10^{16} \mathrm{~m}^{2} / \mathrm{s}^{2}\right)} \geq 4.178 \times 10^{-40} \mathrm{~kg} \text {. }
$$

In reference to the numerical result of equation (5) we must say that in today's standard model the mass values of the elementary particles including that of neutrino with estimated mass are much heavier than the mass predicted above. In table 1, we remind the reader of the order of magnitude of the some of the elementary particle masses.

Table 1. Masses of various elementary particles

\begin{tabular}{|c|c|}
\hline Elementary Particle & Mass $[\mathrm{kg}]$ \\
\hline$m_{e}$ & $9.109 \times 10^{-31}$ \\
\hline$m_{n}$ & $1.674 \times 10^{-27}$ \\
\hline$m_{\mu}$ & $1.883 \times 10^{-28}$ \\
\hline$m_{k+}$ & $8.800 \times 10^{-28}$ \\
\hline$m_{\tau}$ & $3.167 \times 10^{-27}$ \\
\hline$m_{\pi 0}$ & $2.406 \times 10^{-28}$ \\
\hline$m_{\pi+}$ & $2.488 \times 10^{-28}$ \\
\hline$m_{k 0}$ & $8.870 \times 10^{-28}$ \\
\hline
\end{tabular}

Therefore, particles with masses smaller than $m_{0} \leq 10^{-40} \mathrm{~kg}$ will not transform/transmit the information they carry to the universe and therefore as a result they will not be detectable. At this point, we say that Landauer's principle might result in detectability issues that are related to the smallness of the masses of certain elementary particles. In Einstein's theory of general relativity, linearization of Einstein's field equations demonstrates that small perturbations of the metric obey a wave equation (Misner et al. 1973). These small disturbances referred as gravitational waves, and travel at the speed of light. However, 
various gravity theories predict a dispersive propagation (Will and Yunes, 2004). The equation that most commonly describes this behaviour is a Klein -Gordon equation of the form:

$$
\left[\frac{1}{c^{2}} \frac{\partial^{2}}{\partial t^{2}}-\nabla^{2}+\left(\frac{m_{g r} c}{\hbar}\right)^{2}\right] \phi=0
$$

Specifically, the dispersive term is ascribed to the quantum of gravitation having non-zero rest mass $m_{g r}$ or equivalently a non infinite Compton wavelength $\lambda=\hbar / m_{g r} c$ and where $\phi$ is the potential function of the gravitational field. Theories like $M$-theory, loop quantum gravity, string theory and superstring theory and quantum field theory all predict the existence of graviton particles. In relation to quantum field theory gravitons are the elementary particles that mediate the force of gravity, expected to be massless which results to an infinite range gravitational force. Furthermore, gravitons are bosons of spin-2 which is related to the stress energy momentum tensor, and can give rise to a force that is indistinguishable from gravitation simply because the massless spin-2 field is coupled to the stress-energy tensor in the same way that gravity field does (Lightman et al. 1975).

Therefore, if a particle of spin 2 is discovered it must be graviton. Its detection will be an important step in the validation of the gravitational theories above (ibid, 1973) and unify quantum mechanics with general relativity. At the same time, we should say that the extreme weakness of the gravitational force makes the graviton detection a very hard issue. In a recent book by Dyson (2004), the author suggests "the detection of a single graviton may in fact ruled out in the real universe". If this proves to be true, then issues will be raised immediately for the quantization of gravity. On the other hand, attempts to extend the standard model by including gravitons have failed at high energies because of the infinities arising at quantum effects, which means that gravitation is nonrenormalizable (Birrel and Davies, 1994). This also means that quantum mechanics and gravity are incompatible at these energies and thus the situation becomes tenable.

Concluding in relation to the mass of the graviton, we say that in a recent paper by Novello and Neves (2003) the authors indicate a link between the cosmological constant and the graviton mass $m_{g r}$. Theory predicts that particles that travel with the speed of light have practically zero mass $m \geq 10^{-68} \mathrm{~kg}$. This result is in excellent agreement with the current experimental mass bound of photon and graviton, something that suggests that entropic gravity might result of a softly broken recent local symmetry (Mureika and Mann, 2011). Moreover, cosmological holography postulates all the information content in our universe is encoded at the cosmological horizon, a proposal forwarded by Smoot (2010). 


\section{Discussion and Numerical Results}

At this point, we will use Landauer's principle and calculate the corresponding mass of a graviton if the graviton exchanges information with the medium at least one bit of information, using the result in for the temperature of graviton. Thus, according to Evans and Lineweaver (2010) a graviton thermal background is expected to exist which decoupled from the photon bath around the Planck time, and has been cooling since as $T_{g r} \propto \frac{1}{R(t)}$ (ibid 2010). To be more specific Evans and Lineweaver claim that photons cooled less quickly because they have been heated by the annihilation of heavy species, and therefore the current graviton temperature to the current photon temperature and can be written as:

$$
T_{g r}=\left(\frac{g * s\left(t_{0}\right)}{g * s\left(t_{p l}\right)}\right)^{1 / 3} T_{\gamma}
$$

Where: $g_{* s}\left(t_{p l}\right)$ is the relativistic degrees of freedom in the fluid (with $m<k T / \mathrm{c}^{2}$ ) at the Planck time and $g_{* s}\left(t_{0}\right)=3.91$ today and $g_{* s}$ is given by Evans and Lineweaver (2010) as follows:

$$
g_{* s} \cong \sum_{\text {Bosons }_{i}} g_{i}\left(\frac{T_{i}}{T_{\gamma}}\right)^{3}+\sum_{\text {Fermions }_{i}} \frac{7}{8}\left(\frac{T_{j}}{T_{\gamma}}\right)^{3}+\ldots .
$$

In previous estimates of the background graviton entropy the authors have assumed $g_{* s}\left(t_{p l}\right) \sim g_{* s}\left(10^{12} \mathrm{eV}\right)$ $=106.75$ (Frampton \& Kephart 2008) and (Frampton et al. 2009). The corresponding graviton relic temperature as calculated by Evans and Lineweaver (2010) is give by:

$$
T_{g r}=0.61_{-0.52}^{+0.2} \mathrm{~K}
$$

Using the above temperature instead as the temperature under which the graviton now can exchange information and if it exchanges at least one bit of information with the surrounding medium we find that the mass of the graviton to be equal to:

$$
m_{g r} \geq \frac{k_{B} T \ln 2}{c^{2}} \geq 6.483 \times 10^{-41} \mathrm{~kg}
$$

The above-calculated graviton mass exchanging one information bit with the medium is an order of magnitude less than the mass limit predicted by Bormanshenko (2019) where the author has used for temperature of the cosmic microwave background radiation instead. This is the lower bound mass limit required for the graviton to transform information to the medium at its own temperature as calculated by 
Evans and Lineweaver (2010). Therefore, we conclude that at one bit of information gravitons in the range $10^{-69} \mathrm{~kg} \leq m_{g r} \leq 10^{-55} \mathrm{~kg}$ (Nieto and Goldhaber, 2010), will not be detected. As an example, we also say the according to Loureiro et al. (2019) the mass of the lightest neutrino has been estimated to $m_{v}=0.086 \mathrm{eV}=1.5 \times 10^{-37} \mathrm{~kg}$ which is four orders of magnitude heavier that the mass predicted above. Next using the temperature of the graviton (ibid, 2010) we can now write the number of information bits as a function of the graviton mass in the following way (Haranas et al. 2013):

$$
N=\frac{m_{g r} c^{2}}{k_{B} T \ln 2}=1.542 \times 10^{40} m_{g r}
$$

This is the number of information bits, which various graviton masses will be able to transfer to the medium because of their masses. This has been calculated is under the assumption that all gravitons have a temperature equal with that of the graviton relic $T=0.61 \mathrm{~K}$. We can easily see that the number of information bits is a linear function of the mass of the graviton particle in the following way:

$$
N=1.542 \times 10^{40} \mathrm{~m}_{g r}
$$

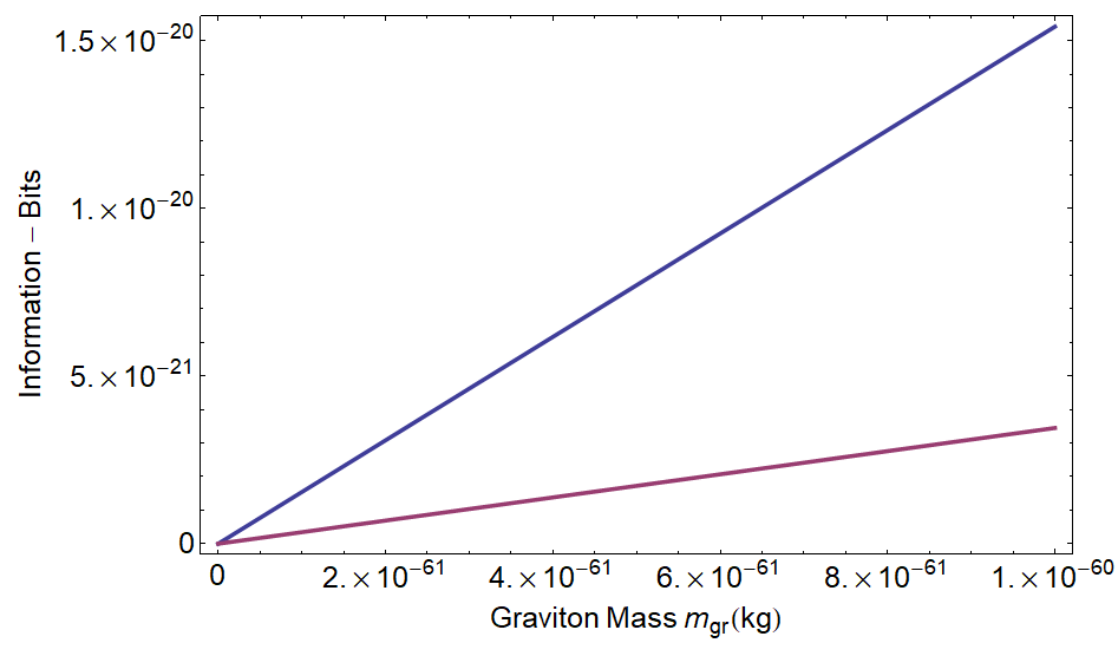

Fig 1:Plot of information bits as function of the graviton mass and for two temperature baths i.e. blue $T=2.725 \mathrm{~K}$ and magenta $T=0.61 \mathrm{~K}$.

Considering the graviton mass range given $10^{-69} \mathrm{~kg} \leq m_{g r} \leq 10^{-55} \mathrm{~kg}$ (Nieto and Goldhaber, 2010) we find that the corresponding number of information contained within the graviton falls in the range:

$$
1.542 \times 10^{-29} \text { bits } \leq N \leq 1.542 \times 10^{-15} \text { bits } .
$$


From equation (12) we see that it takes a quantifiable graviton of mass $m_{g r}=6.485 \times 10^{-41} \mathrm{~kg}$ to contain and transfer one bit of information to the medium, which is impossible since the calculated graviton mass range is many orders of magnitudes smaller that the limiting mass. In our calculation, we have used the corresponding graviton temperature as calculated by Evans and Lineweaver (2010), but the mas is still many orders of magnitude when compared with that mass of most elementary particles as given in table 1 above. In reference to Eq. (13) in Haranas and Gkigkitzis (2013), certain cosmological scenarios involve fractional number of information bits. According to information theory, fractional information bits imply uncompressed data. This will also imply information that is not possible to be decompressed (or sometimes it has not been decompressed yet) through matter and energy. Thus, we may perceive matter in our universe as a system of specific information in which any interaction and law takes place with the exchange of specific amount of information under the assumption that matter is an entity analogous to a computer.

Next, is known that the Compton wavelength or range of a boson $\lambda$ in this case the graviton it is given by the following relation (Haranas, 2017):

$$
\lambda_{g r}=\frac{\hbar}{m_{g r} c} .
$$

Using Eq. (3) for or the mass of graviton, as function of the maximum number of information $N$ that may be recorded by the particle with the bath, we can write the range of graviton in the following way:

$$
\lambda_{g r}=\frac{\hbar c}{N k_{B} T \ln 2} \propto \frac{1}{N} .
$$

In other words, the range of the graviton scales inversely proportional to the information number $N$. Thus, if a graviton contains a very small number of information bits is expected to have a very large range or Compton wavelength. In that aspect we postulate that the less information a graviton carries the less will probably interact with the rest of the universe and it will propagate in large cosmic distances. Therefore, we propose that the information bit might be an alternative way via which graviton, particles and matter could interact with the rest of the universe. Thus, we can picture information as a new way via which matter interacts with the rest of the universe. It could be that information also tells matter how to interact. Using the values predicted for the mass of graviton according to Nieto and Goldhaber (2010) we find that $\lambda$ falls in the range:

$$
3.513 \times 10^{26} \mathrm{~m} \leq \lambda \leq 3.513 \times 10^{12} \mathrm{~m} .
$$


Talking into account that the radius of the universe is $R_{u n i}=4.4 \times 10^{26} \mathrm{~m}$ (Bars and Teming, 2009) and (Davis and Lineweaver 2004) we say that such a graviton of different masses can have a ranges approximately equal to the size of the visible universe and the solar system respectively. In Finn and Sutton (2002) applied this idea in a investigation of the data from the Hulse-Taylor binary pulsar and from the pulsar PSR B1534+12. From their analysis of the data, they found the graviton range to be $\lambda \geq 2.60 \times 10^{12} \mathrm{~m}$ which is almost identical to our lower bound in equation (16). That was under the assumption that, some linearized theories, allow a massive graviton which would propagate freely via the Klein-Gordon equation of a particle with mass $m_{g r}$. If the graviton had a rest mass, the decay rate of an orbiting binary would be affected (Taylor et al., 1992). As the decay rates of binary pulsars agree very well with GR, the errors in their agreements provide a limit on a graviton mass.

Next, in a more general way following Viaggiu (2017) we can write the entropy $S_{g r}$ in an enclosure (box) in the following way:

$$
S_{g r}=-k_{B} N_{g r_{i}}\left[\ln \left(1-e^{-x / 2}\right)+\ln \left(1-e^{-x}\right)\right]+\frac{\pi c \hbar N_{g r_{i}}}{2 T R} e^{-x / 2} \frac{\left(1+3 e^{-x / 2}\right)}{\left(1-e^{-x}\right)} .
$$

where:

$$
x=\frac{\pi c \hbar}{k_{B} T R}
$$

where again $R$ is the dimension of the "box" and is $N_{g r_{i}}$ the number of gravitons enclosed. Taking equation (18) to the horizon i.e. $R_{H}=c / H$ we obtain that:

$$
x=\frac{\pi \hbar H}{k_{B} T} .
$$

In the present era $x<<1$ has an extremely small value and therefore expanding (17) to first order we obtain that:

$$
S_{g r} \cong\left[\frac{2 \pi c \hbar}{R T x}+\left(\frac{3 k_{B}}{4}+\frac{5 \pi c \hbar}{48 R T}\right) x+\left(k_{B} \ln \left(\frac{2}{x^{2}}\right)-\frac{3 \pi c \hbar}{4 R T}\right)\right] N_{g r_{i}} .
$$

Using the value of $x$ and after some algebra we can write eq. (20) in the following form:

$$
S_{g r} \cong 2 k_{B}\left[1+\frac{5}{96} x^{2}+\frac{1}{2} \ln \left(\frac{2}{x^{2}}\right)\right] N_{g r_{i}},
$$

for which we can say that the entropy for a total number of gravitons $N_{g r_{i}}$ treating gravitons as harmonic oscillators in an enclosure of size $R$ and temperature $T$ and it's equal to an effective $k_{\mathrm{B}}$ Boltzmann constant, by twice the value of the square bracket in the above equation. It is the proportionality factor that relates the average relative kinetic energy of the gravitons in the graviton gas with the thermodynamic temperature of the 
gas. Equating Eq. (20) to the equation of the entropy of a system in terms of the information number $N$ i.e. $S=N_{t o t} k_{B} \ln 2$ and solving for the number of information $N$ corresponding to an number of gravitons $N_{g r_{i}}$ we obtain that:

$$
N_{t o t} \cong \frac{2}{\ln 2}\left[1+\frac{5}{96} x^{2}+\frac{1}{2} \ln \left(\frac{2}{x^{2}}\right)\right] N_{g_{i}} .
$$

Taking $R$ to be the Hubble radius $R_{H}=c / H_{0}$ and assuming one graviton $N_{i}=1$. equation (21) after some simplifications takes the form:

$$
N=\frac{2}{\ln 2}\left[1+\left(\frac{\pi \hbar H_{0}}{k_{B} T}\right)^{2}+\frac{1}{2} \ln \left(2\left(\frac{k_{B} T}{\pi \hbar H_{o}}\right)^{2}\right)\right] .
$$

Next, substituting Eq. (22) into (15) we can write the range $\lambda_{g r}$ of one graviton in the following way:

$$
\lambda_{g r}=\frac{\hbar c}{k_{B} T \ln 2\left(\frac{2}{\ln 2}\left[1+\left(\frac{\pi \hbar H_{0}}{k_{B} T}\right)^{2}+\frac{1}{2} \ln \left(2\left(\frac{k_{B} T}{\pi \hbar H_{o}}\right)^{2}\right)\right]\right)} .
$$

Given that in the present era the second term in the denominator square bracket is $\left(\frac{\pi \hbar H_{0}}{k_{B} T}\right)^{2}<<1$ equation (23) can be written as:

$$
\lambda_{g r}=\frac{\hbar c}{2 k_{B} T\left(1+\frac{1}{2} \ln \left(2\left(\frac{\pi \hbar H}{2 k_{B} T}\right)^{2}\right)\right)} .
$$

For one graviton that carries at least one bit of information to have an infinite range would be possible if the temperature of the thermal bath is equal to:

$$
T=\frac{\pi \hbar H}{k_{B} e \sqrt{2}}=\frac{\pi}{e \sqrt{2}}\left(\frac{\hbar H}{k_{B}}\right)=\frac{\pi}{e \sqrt{2}}\left(\frac{E_{\text {min }_{\text {qua }}}}{k_{B}}\right),
$$

where we have used that $E_{\text {min }_{\text {qua }}}=\hbar H$ is the so-called minimum quantum energy of the graviton (Gershtein et al. 2003). Next, let us now consider the total number of gravitons in the universe as its given in Haranas and Gkigkitzis (2014):

$$
N_{i}(g r)=\frac{c^{3}}{G \hbar \Lambda}=\frac{1}{\ell_{p l}^{2} \Lambda},
$$


where $\Lambda$ is the cosmological and $\ell_{p l}$ is the Planck length. Substituting (27) in (15) we obtain the total number of information for the total number of gravitons in the universe is equal to:

$$
N_{\text {tot }} \cong\left(\frac{1}{\ell_{p l}^{2} \Lambda}\right)\left[1+\frac{1}{2} \ln \left(2\left(\frac{k_{B} T}{\pi \hbar H_{0}}\right)^{2}\right)\right] \text {. }
$$

Using eq. (27) we find an average graviton range to be equal to:

$$
\left\langle\lambda_{g r}\right\rangle=\frac{\ell_{p l}^{2} \Lambda}{2 k_{B} T} \frac{\hbar c}{\left.1+\frac{1}{2} \ln \left(2\left(\frac{k_{B} T}{\pi \hbar H_{0}}\right)^{2}\right)\right]} .
$$

Next let us assume that the graviton can have an "infinite" range, would imply that the denominator of eq. (28) should be equal to zero for two different possibilities for the thermal bath temperature, i.e. $T=0$ and also $T$ equal to equation (25) as before

$$
T=\frac{\pi \hbar H}{k_{B} e \sqrt{2}}
$$

Similarly, and infinite range will require a Hubble constant that is equal to:

$$
H=\frac{e \sqrt{2}}{\pi}\left(\frac{k_{B} T}{\hbar}\right)
$$

In flat universe where the Hubble constant depends on information in the following way (Haranas et al. 2013a)

$$
H=\sqrt{\frac{\pi}{\ln 2}} \frac{1}{t_{p} \sqrt{N}} .
$$

Equating (30) and (31) we can write this temperature of the bath as a function of information in the following way: (34)

$$
T=\frac{\pi^{3 / 2}}{e \sqrt{\ln 4}}\left(\frac{\hbar}{k_{B} t_{p l} \sqrt{N}}\right),
$$

where $t_{p l}=\sqrt{\frac{G \hbar}{c^{5}}}$ is the Planck time, and therefore equation (32) can be further written as follows:

$$
T=\frac{\pi^{3 / 2}}{e \sqrt{\ln 4}}\left(\frac{T_{p l}}{\sqrt{N}}\right)
$$

where $T_{p l}=\sqrt{\frac{\hbar c^{5}}{G k_{B}^{2}}}=1.416 \times 10^{32} \mathrm{~K}$ is the Planck temperature. At this point considering the universe an enclosure of the size of today's horizon we calculate its total entropy $S_{t o t}$. Following Viaggiu (2017) and 
treating gravitons as harmonic oscillators and using that entropy is equal to $S_{t o t}=N_{t o t} k_{B} \ln 2$, (Haranas and Gkigkitzis 2013b) and substituting eq (26) for the total number of gravitons in the universe we find that:

$$
S_{t o t}=2\left(\frac{k_{B} c^{3}}{G \hbar \Lambda}\right)\left[1+\frac{1}{2} \ln \left(\frac{k_{B} T}{\pi \hbar H_{0}}\right)^{2}\right]=\frac{2 k_{B}}{\ell_{p l}^{2} \Lambda}\left[1+\frac{1}{2} \ln \left(2\left(\frac{E_{t h r}}{E_{q u_{m i n}}}\right)^{2}\right)\right]
$$

Calculating equation (34) in the present era so we find that the total number of information is:

$$
N_{\text {tot }}=67.42\left(\frac{k_{B} c^{3}}{G h \Lambda}\right)=\frac{67.42}{\ell_{p}^{2} \Lambda}=0.675 \times 10^{99} \text { nat }\left(\frac{1}{\ln 2}\right) \text { bits } / \text { nat }=0.972 \times 10^{99} \text { bits }
$$

The above entropy is approximately 0.271 times smaller than the one calculated by Egan and Lineweaver (2010) at the visible horizon of the universe, namely $S_{h o r}=2.60 \times 10^{122} k_{B}=3.588 \times 10^{99} \mathrm{~J} / \mathrm{K}$. The reason for that is simply the contribution of an extra term that enters the calculation of the graviton entropy, in a first order approximation in an enclosure of dimension $R$ in the treatment of Vaggiu (2017), when gravitons are treated as harmonic oscillators. In this case if we use equation (40) in Egan and Lineweaver (2017) paper namely:

$$
S_{H}=\frac{k_{B} c^{3} A_{H}}{4 G \hbar},
$$

the radius of the horizon and solving for the radius of the horizon we obtain:

$$
R_{U}=\left[\frac{2}{\pi \Lambda}\left(1+\frac{1}{2} \ln \left(\frac{2 k_{B}^{2} T^{2}}{\pi^{2} \hbar^{2} H_{0}^{2}}\right)\right)\right]^{1 / 2}
$$

where today's radius of the universe is calculated using the black body background radiation temperature $T=2.725 \mathrm{~K}$ and also the individual graviton temperature as given by Egan and Lineweaver (2010) i.e. $T=$ $0.61 \mathrm{~K}$ in the above equation we find that:

$$
\begin{aligned}
& R_{U}=6.161 \times 10^{26} \mathrm{~m}, \\
& R_{U}=6.230 \times 10^{26} \mathrm{~m},
\end{aligned}
$$

where the radius of the universe in Egan and Lineweaver is calculated to be $R_{u}=1.550 \times 10^{26} \mathrm{~m}$ (ibid, 2010). At his point, we estimate the temperature required for which the graviton has an infinite range. In the present era we find that the corresponding temperature is:

$$
T_{\lambda_{g r \rightarrow \infty}}=1.433 \times 10^{-29} \mathrm{~K} .
$$


This is an extremely low temperature. To obtain an idea of such a low temperature let us consider the mass universe of $M_{U}=\frac{c^{3}}{G H_{0}}=1.762 \times 10^{53} \mathrm{~kg}$ (Haranas and Gkigkitzis, 2013b), to be a black hole and calculate the temperature of such black hole to be:

$$
T_{B H}=\frac{\hbar c^{3}}{8 \pi k_{B} G M_{U}}=6.980 \times 10^{-31} \mathrm{~K} .
$$

Similarly using equation (15) and solving for the number of information $N$ we obtain that:

$$
N=\frac{\hbar c}{\lambda_{g r} k_{B} T \ln 2} .
$$

Assuming that the range of the graviton is at least equal to the visible horizon of the universe i.e.

$$
\begin{array}{r}
\lambda_{g r}=R_{U}=\frac{c}{H_{0}} \text { equation (42) becomes } \\
N=\frac{\hbar H_{0}}{k_{B} T \ln 2}=\frac{1}{\ln 2}\left(\frac{E_{\text {qua } \min }}{E_{\text {ther }}}\right) .
\end{array}
$$

Using the temperature of microwave background radiation i.e. $T=2.725 \mathrm{~K}$ as well as the temperature of the graviton relic as given in equation (9) $T=0.61 \mathrm{~K}$ (Evans and Lineweaver 2010) equation (42) gives the following values for the information $N$ :

$$
9.294 \times 10^{-30} \text { nats } \leq N \leq 4.152 \times 10^{-29} \text { nats . }
$$

At this point we say that $N=O\left(10^{-29}\right)$ is the same order of magnitude of information as given in equation (12) and for $m_{g r} \geq 10^{-69} \mathrm{~kg}$, which means either by mass or by a relic graviton temperature $T=0.61 \mathrm{~K}$ the same amount of information can be included. Thus, we say that the graviton has two distinct possibilities in achieving the same amount of information. Using equation. (33) and the numerical value of the temperature of the thermal bath we estimate the amount of information in one single graviton to be equal to:

$$
N=\frac{\pi^{3}}{2 e^{2}(\ln 4)}\left(\frac{T_{p l}}{T_{\lambda_{g r \rightarrow \infty}}}\right)^{2}=2.132 \times 10^{122} \text { nats. }
$$

This number is the same order of magnitude as the total number of gravitons in the universe being equal to:

$$
N_{g r}(\text { tot })=3.626 \times 10^{121} \text {. }
$$

With reference to Haranas and Gkigkitzis (2014) the authors have derived an expression for the total number of gravitons contained inside the observable horizon $N_{g r_{t o t}}$ as a function of the number of information $N$ in the following way: 


$$
N_{g r_{t o t}}=\frac{\ln 2}{3 \pi} N=0.073 N
$$

where $N$ is the number of the total information in the universe. Looking at equation (45) it might be worth trying to understand this numerical result. We postulate that the result might be related to signal processing, data compression, and source coding, or bit-rate reduction which is the process of encoding information by making use of fewer bits that the original representation. This is known as lossless compression and reduces the number of bits by identifying and eliminating the statistical redundancy but without any information lost during the process, which is an algorithmic one. This is taking place by removing unnecessary or unimportant information. In our case we postulate that the universe performs data compression, playing the role of an encoder, and the graviton which performs the decompression plays the role of a decoder. Such a compression perhaps uses the spacetime physics and geometry as resources required to store and transmit data. Computational resources such as history of orbits, interactions and configurations are consumed in the compression and decompression processes. Data compression is subject to a space-time complexity trade-off. The design of data compression schemes involves trade-offs among various factors, including the degree of compression, the amount of distortion introduced (when using lossy data compression, perhaps due to an increasing entropy in the universe), and the computational resources required to compress and decompress the data.

For example, highly repetitive input such as the orbit of a planet or particle can effectively be compressed, for instance, such as in biology a biological data collection of the same or closely related species can be compressed. The basic task can be achieved through a context free physics. However, just as the human eye is more sensitive to subtle variations in luminance than it is to the variations in color, it is very likely that our perception is restricted to the use of several compression formats that lead to paradoxes, such as a graviton having information context greater than the universe.

Inspired by the close connection between machine learning and compression where a system can predict the posterior probabilities of a sequence given its entire history for optimal data compression, we may expect that a particle such as the graviton, can read the history of the universe and has more bits of information as predicted posterior probabilities of the system (the concept of "general intelligence. (Mahoney, 2013), (Shmilovici et al., 2009) and (Ben Gal 2008). When we study the universe, our rational perception resembles the psychoacoustics of our ears where not all data in an audio stream can be perceived by the human auditory system. But as a lossy compression, it reduces redundancy by first identifying perceptually irrelevant sounds, that is, sounds that are very hard to hear.

Another hypothesis we can make is that perhaps the universe manifests only certain aspects of its information context that can be measured or identified by our scientific methods. Something like it happens to speech encoding, an important category of audio data compression. The range of frequencies needed to 
convey the sounds of a human voice are normally far narrower than that needed for music, and the sound is normally less complex. As a result, speech can be encoded at high quality using a relatively low bit rate. Perhaps the information context of the universe has an intrinsic mechanism that "hides" away more of the information bits-keeping just enough to reconstruct an "intelligible" universe to the observer rather than the full realization range of events, that can be modeled even beyond quantum probabilities.

\section{Conclusions}

In this paper the idea that the max number of information $N$ recorded by one particle emerged in a heat bath is inversely proportional to the particle's temperature $T$. Furthermore if at least one bit of information it is transferred from the particle to the medium, then the corresponding particle might exchange information with the medium. If the particle exists in our universe and the temperature to be the same of that of black body radiation $T=2.73 \mathrm{~K}$, the corresponding mass of the particle carrying at least one bit of information is equal $m=4.718 \times 10^{-40} \mathrm{~kg}$ which is many orders of magnitude smaller that the masse of most of today's elementary particles. Next, using the corresponding temperature of a graviton i.e. $T_{g r}=0.61_{-0.52}^{+0.2}$ and assuming at least for one bit of information the corresponding graviton mass is calculated and from that, a relation for the number of information $N$ carried by a graviton as a function of the graviton mass $m_{g r}$ is derived. Furthermore, the range of information number contained in a graviton is also calculated for the given range of graviton mass as given by Nieto and Goldhaber and, we find that the range of the graviton is inversely proportional to the information number $N$. Finally, treating gravitons as harmonic oscillators in an enclosure of size $R$ we derive the graviton range as a function of cosmological constant, graviton temperature and the Hubble parameter.

\section{References}

Bars I., and Teming J., Extra Dimensions in Space and Time, Springer, (2009) New-York, NY, USA

Ben-Gal, On the use of data compression measures to analyze robust designs, IEEE Transactions on Reliability, (2008), 54 (3), 381-388.

Birrel N., D., P. C., Davies, Quantum fields in curved space, Cambridge Monographs on Mathematical Physics, p. 150, 1994.

Bormashenko E. The Landauer Principle: Re-Formulation of the Second Thermodynamics Law or a Step to Great Unification? Entropy (2019), 21, 918, doi:10.3390/e21100918. 
Davis M., and Lineweaver C., "Expanding confusion: common misconceptions of cosmological horizons and the superluminal expansion of the universe," Publications of the Astronomical Society of Australia, (2004) vol.21, no.1, pp.97-109.

Dyson, F., J., "The world on a string", (2004) review of The Fabric of the Cosmos: Space, Time, and the Texture of Reality by Brian Greene, NY Rev. Books 51(8).

Egan, C.A., Lineweaver, C.H.: A larger estimate of the entropy of the Universe. Astrophys. (2010) J. 710 (2), 1831.

Finn L. S. and Sutton P. J., "Bounding the Mass of the Graviton Using Binary Pulsar Observations, (2002) Phys. Rev. D 65, 044022/1-7.

Frampton, P. H. \& Kephart, T. W. (2008), Journal of Cosmology and Astro-Particle Physics, 6, 8

Frampton, P. H., Hsu, S. D. H., Kephart, T. W., \& Reeb, D., Classical and Quantum Gravity, 2009, 26, 145005 .

Gershtein, S. S. A. A. Logunov, M. A. Mestvirishvili, Graviton mass and total relative density of mass $\Omega_{t o t}$ in Universe, Dokl.Phys.48:282-284,2003; Dokl. Akad. Nauk Ser. Fiz.48: 755-757.

Goldhaber, A S. and Nieto M M, Photon and Graviton Mass Limits, Rev. of Mod. Phys. 82, 932, 2010

Haranas I., Gkigkitzis I., Kirk S., (2013a) Number of information and its relation to the cosmological constant resulting from Landauer's principle, Astrophys Space Sci, 348, pages553-557.

Haranas, I., Gkigkitzis, I.: Bekestein bound of information number $N$ and its relation to cosmological parameters in a Universe with and without cosmological constant. Mod. Phys. Lett. A (2013b). 28(19), 1350077.

Haranas, I., and Gkigkitzis, I.: The number of information bits related to the minimum quantum and gravitational masses in a vacuum dominated universe. Astrophys. Space Sci. (2013). doi:10.1007/ s10509013-1434-1.

Haranas I., and Gkigkitzis I., The Mass of Graviton and Its Relation to the Number of Information according to the Holographic Principle, (2014) Hindawi Publishing Corporation International Scholarly Research Notices, Volume 2014, Article ID 718251, 8 pages.

A. P. Lightman, W. H. Press, R. H. Price, and S. A. Teukolsky, Problem 12.16. Problem Book in Relativity and Gravitation, Princeton University Press, 1975

Landauer, R. Disisipation of heat generation in the computing process. IBM Journal of Research and Development (1961) 5, 183.

Landauer, R.: Dissipation and noise immunity in computation and communication. Nature (1998) 335, 779.

Lloyd, S.: Ultimate physical limits to computation. Nature (2000) 406, 1047- 1054.

Lloyd,S.: Computational capacity of the Universe. Phys.Rev.Lett. (2002), 88, 237901. 
Mahoney M., Rationale for a large text compression benchmark, Florida Institute of Technology, Retrieved 5 March 2013.

J. R. Mureika J. R., and R. B. Mann, "Does entropic gravity bound the masses of the photon and graviton?" (2011) Modern Physics Letters A, vol. 26, no. 3, pp. 171-181.

Goldhaber A. S., and Nieto M. M., Photon and graviton mass limits, (2010) Rev. Mod. Phys. 82, 939.

Norton, J.D.: Eaters of the lotus: Landauer's principle and the return of Maxwell's demon (2004). http://philsci-archive.pitt.edu/1729/

M. Novello and R. P. Neves, "The mass of the graviton and the cosmological constant," Classical and Quantum Gravity, (2003) vol. 20, no. 6, pp. L67-L73.

Shenker, R.: Logic and Entropy (2000). http://philsci-archive.pitt.edu/ id/eprint/115.

Shmilovici A., Kahir Y., Ben-Gal I., Hauser S., (2009) Measuring the efficiency of the intraday forex market with universal data compression algorithm, Computational Economics, 33(2) 131-154.

Smoot G. F., Go with the Flow, Average Holographic Universe, (2010) Int. J. Mod. Phys. D19: 2247-2258.

Taylor, J. H., A. Wolszczan, T. Damour, and J. M. Weisberg, (1992) “Experimental Constraints on StrongField Relativistic Gravity,” Nature 355, 132-136.

Valev, D.: Estimations of total mass and energy of the universe (2010). arXiv: 1004.1035 [physics.gen-ph]. Viaggiu S., Statistical mechanics of gravitons in a box and the black hole entropy, (2017) Physica A 473 412-422.

Vopson M., The mass-energy-information equivalence principle, (2019) AIP Advances, 9, 095206; doi: 10.1063/1.5123794

Wesson, P.S.: Is mass quantized (2003). arXiv:gr-qc/0309100 v1. 20. 